\title{
SYNTHESIS AND MORPHOLOGY OF SILICON NANOPARTICLES BY DEPOSITION TIME VARYING LPCVD METHOD TO DEMONSTRATE THE VARIATION OF HEIGHT, DENSITY AND SIZE
}

\author{
Mitra Barun Sarkar ${ }^{1}$, Joydeep Datta ${ }^{2}$, Diptojyoti Mondal ${ }^{3}$, Sabyasachi Mukhopadhyay ${ }^{4}$ \\ ${ }^{1}$ Assistant Professor, Electronics and Communication Engineering, NIT Agartala, Tripura, India, barun06@gmail.com \\ ${ }^{2}$ U.G.Student, Electronics and Communication Engineering, RCCIIT, West Bengal, India, joydeep.datta100@gmail.com \\ ${ }^{3}$ U.G.Student, Electronics and Communication Engineering, RCCIIT, West Bengal, India, mondal.diptojyoti@gmail.com \\ ${ }^{4}$ Research Fellow, Physical Sciences Department, IISER-K, West Bengal, India, mukhopadhyaysabysachi@gmail.com
}

\begin{abstract}
The confinement of the modern day semiconductor devices is fast propelling the growth of nanoparticles as an excellent constituent of such device and the characteristics like quantum size confinement and coulomb blockade effect of the same is accelerating their application in this field. But before the fabrication of devices with the nanoparticles, their morphological and electrical characteristics needs to be studied in order to have a sense about the efficiency of such a device. In this experiment, Silicon nanoparticles have been deposited on $\mathrm{SiO}_{2}$ substrate by thermal decomposition of silane using Low Pressure Chemical Vapor Deposition (LPCVD) Method with varying deposition times. Then, the morphology of the sample, the size as well as distribution of the nanoparticles is investigated using Atomic Force Microscopy (AFM) and the Scanning Probe Image Processor (SPIP). It shows that all the characteristics of the nanoparticles like height, density and size of the nanoparticles change with the varying deposition time. The size of the nanoparticles varied between 2-10nm whereas the height and density varied between $1-3 \mathrm{~nm}^{\mathrm{and}} 2 \times 10^{11} / \mathrm{cm}^{2}$ $3.5 \times 10^{11} / \mathrm{cm}^{2}$ respectively.
\end{abstract}

Index Terms: Silicon Nanoparticles, Nucleation, Quantum Confinement, LPCVD, AFM etc.

\section{INTROUCTION}

The dimensions of semiconductor devices are shrinking fast day by day which is actually propelling the advancement of modern day computers and gadgets. The confinement of dimensions has already reached into nanoscale range and attempts are going on to prepare such in order to decrease the power consumption and dissipation when they are applied in a device. When such a miniaturization is continued, it becomes increasingly difficult to fabricate the device and squeeze out its properties. It is the case where the quantum size plays an important role.

Quantum size confinement, Coulomb Blockade effect etc. makes silicon nanocrystals a possible candidate for the fabrication of novel devices such as single electron transistors [1], vertical transistors [2], resonant tunneling devices [3-4] and floating gate memory devices [5-8]. The quantum confinement effect is observed when the size of the particle is too small to be comparable to the wavelength of the electron. So as the size of a particle decreases till nano-scale range, the decrease in confining dimension makes the energy levels discrete and this increases or widens up the band gap and ultimately the band gap energy also increases. Since the band gap and wavelength are inversely related to each other the wavelength decrease with decrease in size and the proof is the emission of blue radiation.

Nanoparticles can be produces by LPCVD, PECVD and laser ablation. However, there is little research in the way of the particle electrical characteristics. In general, this has involved building a composite structure of nanoparticles embedded in an insulating matrix [9] or measuring the electrical properties of freestanding nanoparticles by making two direct electrical contacts using Scanning Tunneling Microscopy [10-11] or atomic force microscopy [3]. Due to the strong carrier confinement in a silicon nanoparticle, it is reasonable to assume that the electrical properties of these particles will be different from their thin film equivalents. For example, the current through a nanoparticle can vary by several orders of magnitude when the addition or removal of a single electron changes the charge on a nanoparticle. Since the location of nanoparticles is random, a critical step in nanoparticle device fabrication is the exploration of electrical properties of the nanoparticles.

The silicon nanoparticles used in this study were deposited on $\mathrm{SiO} 2$ substrate by thermal decomposition of silane using Low Pressure Chemical Vapor Deposition (LPCVD) [12-16] and is 
investigated under different time frames. Varying the time, temperature and partial pressure of silane gas can change the average diameter and height of the hemispherical nanoparticles formed. This route offers an excellent control over particle size and size distribution and particle density.

In this paper, synthesis of silicon nanoparticles is going to be described by low-pressure chemical vapor deposition (LPCVD) [4] method and is investigated under different time frames. Samples' morphology, nanoparticles' size and distribution have been studied by Atomic Force Microscope (AFM) and image processing software Scanning Probe Image Processing Software(SPIP).

\section{EXPERIMENTAL DETAILS OF SYNTHESIS OF NANOPARTICLES}

The device fabrication process begins with the<100> orientation n-type silicon wafer $(9-11 \Omega \mathrm{cm})$ of 2" diameter after various type of cleaning. Contaminants present on the surface of silicon wafers at the start of processing, or accumulated during processing, have to be removed at specific processing steps in order to obtain high performance and highly reliable semiconductor devices.

In the surface wafer cleaning process, silicon wafer was boiled in tri-ethylene for 5 minutes to remove glycerin and wax in the wafer. Then the wafer was dipped in methanol for few minutes to remove dust and fragmented particles and rinsed for 1minute in running DI water. The RCA cleaning is followed by Piranha cleaning.

The Piranha cleaning mixture is combined of sulfuric acid $\left(\mathrm{H}_{2} \mathrm{SO}_{4}\right)$ and hydrogen peroxide $\left(\mathrm{H}_{2} \mathrm{O}_{2}\right)$, usually $3: 1$ in volume ratio to remove organic and metallic impurities. It is the most effective in cleaning silicon surface. Many cleaning steps include an HF last step to remove native oxide and after that, the following procedure was maintained.

In the next dry thermal oxidation process, $\mathrm{SiO}_{2}$ has been grown on $\mathrm{Si}$ substrata at $800^{\circ} \mathrm{C}$ for 5 minutes in the presence of $\mathrm{O}_{2}$. THERMCO oxidation furnace has been used in this process. Thus, $50 \AA \mathrm{SiO}_{2}$ layer (tunneling oxide) was grown by thermal oxidation on $n$ type $<100>$ silicon substrate. The chemical formulation is as follows:

$$
\mathrm{Si}+\mathrm{O}_{2} \rightarrow \mathrm{SiO}_{2} \quad\left(800^{\circ} \mathrm{C}\right)
$$

Next, the silicon nanoparticles have been deposited using LPCVD method. In LPCVD method, Silicon is deposited by pyrolysing silane at $600^{\circ} \mathrm{C}$ in a low-pressure THERMCO CVD reactor. Here the chemical formulation goes like this:

$$
\mathrm{SiH}_{4} \rightarrow \mathrm{Si}+2 \mathrm{H}_{2}\left(600^{\circ} \mathrm{C}\right)
$$

In this process, the deposition parameters like as Process Temperature (600C), Chamber Pressure (100 mTorr) and Silane Flow Rate $(50 \mathrm{sccm})$ and Time (varied between: 15 sec- $90 \mathrm{sec}$ ) have been considered exclusively for our experiment.

\section{AFM CHARACTERIZATION PROCEDURES}

Nanodimensional structures are characterized by scanning probe microscopy (SPM) techniques like AFM, scanning tunneling microscopy (STM). There are different modes of AFM imaging, namely contact, non-contact, intermittent contact (or tapping), and lateral force microscopy. Contact mode is the most common mode of operation of the AFM. In this mode, the tip and the sample remain in close contact (in the repulsive regime of the intermolecular force curve). One of the drawbacks of contact mode is that there exist large lateral forces on the sample as the tip is dragged over the specimen and may damage the sample of soft material like biological or polymer specimen. In non-contact mode, cantilever oscillates above the surface of the sample at such a distance that it is not in the repulsive force regime. This is a very difficult mode to operate in ambient conditions. The water meniscus, which exists on the surface on the sample, invariably forms a small capillary bridge between the tip and the sample and causes the tip to jump-to-contact. Tapping mode is the next most common mode used in AFM. When operated in air or other gases, the cantilever is oscillated at its resonant frequency (hundreds of $\mathrm{kHz}$ ) and is positioned above the surface so that it only taps the surface for a very small duration. As a result, lateral forces are significantly reduced as the tip scans over the surface. When imaging poorly immobilized or soft samples, tapping mode is a better choice than the contact mode. For this study, (nanosilicon/SiO2/Si samples), the contact mode AFM imaging is employed as the sample material is hard and had gone through various high temperature cycles during fabrication. Digital Instrument AFM was used with scanner size of $1 \mathrm{~mm}$.

The AFM images have been analyzed using available software namely SPIP. These software packages offer a wide variety of functions related to AFM image analysis including crosssection profile analysis, roughness analysis, grain/pore analysis, 3-D visualization, plane correction (flattening)etc. Line profiles were employed to estimate size and height of nanoparticles. Nanoparticle density has been calculated by counting particles in the window using the image processor.

\section{CHARACTERIZATION OF SURFACE MORPHOLOGY by ATOMIC FORCE MICROSCOPE (DIGITAL INSTRUMENT) \& SPIP}

Si nanoparticles were deposited at temperature 6000C and at chamber pressure $100 \mathrm{mT}$ Torr. The variation of deposition time was between 30-120sec. The surface morphology 
characterization was done by Atomic Force Microscope (AFM). The AFM image of nanoparticles after 30 secs of deposition time has been shown below:

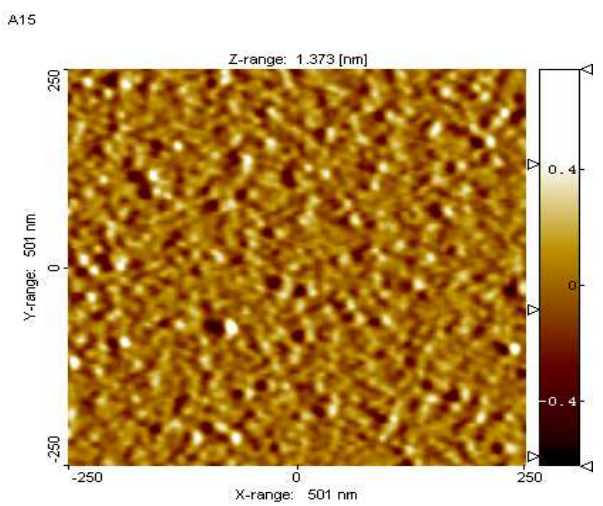

Fig. 1: AFM image of nanoparticles deposited at $600 \square \mathrm{C}$ for $30 \mathrm{sec}$

Images have been analyzed by SPIP (Scanning Probe Image Processor, SPIP, (OImage Metrology A/S) software which has provided the following result. Using SPIP, we have got the $3 \mathrm{~d}$ image window that elucidated the shape of the nanoparticles and the density of nanoparticles. The AFM image of nanoparticles after deposition of 90 secs is shown below:

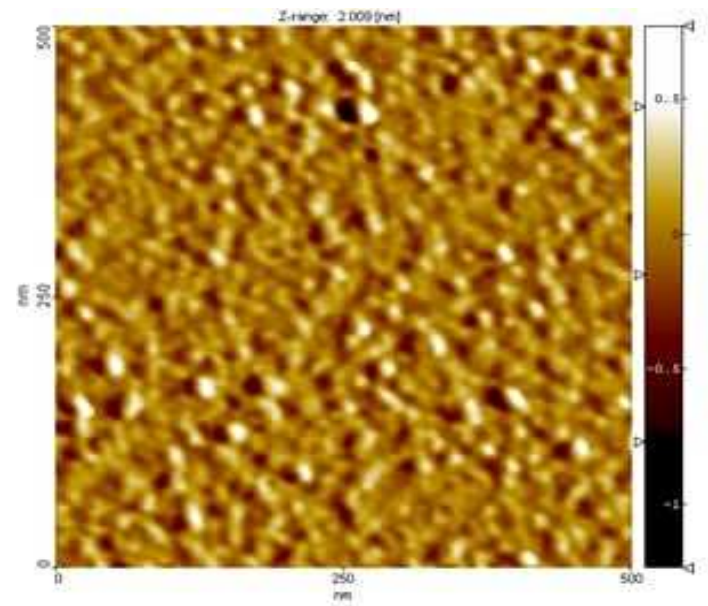

Fig. 2: AFM image of nanoparticles deposited at $600^{\circ} \mathrm{C}$ for $90 \mathrm{sec}$

The line profiles of the same sample of Fig. 2 have been shown below.

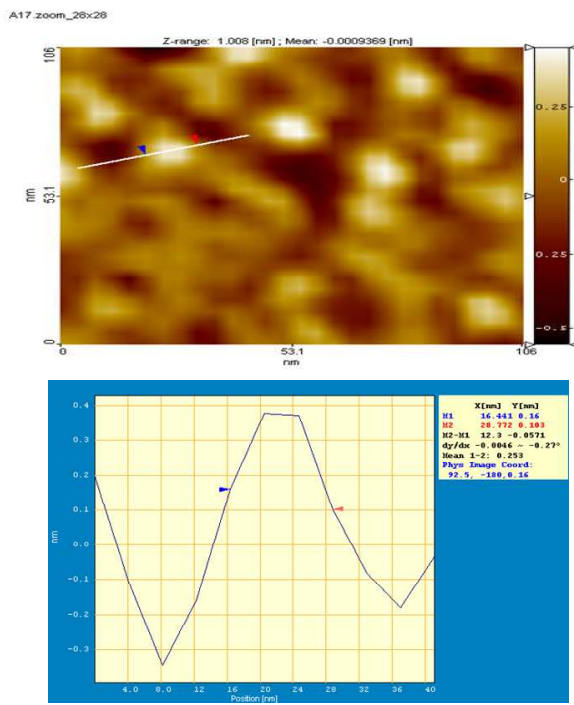

Fig3: Line Profile of nanoparticles deposited at $6000 \mathrm{C}$ by LPCVD process for $90 \mathrm{sec}$

From this image, a small window of size $500 * 500 \mathrm{~nm}$ have been extracted for analyzing the particles size, number and distribution using SPIP (Scanning Probe Image Processor, SPIP, (OImage Metrology) software. Using SPIP we get the 3$\mathrm{D}$ image window that elucidated the size of the nanoparticles in the range of 2-10 $\mathrm{nm}$ and density: $2 \times 1011 / \mathrm{cm} 2$. The following Fig. 3 is the 3D image of the window extracted:

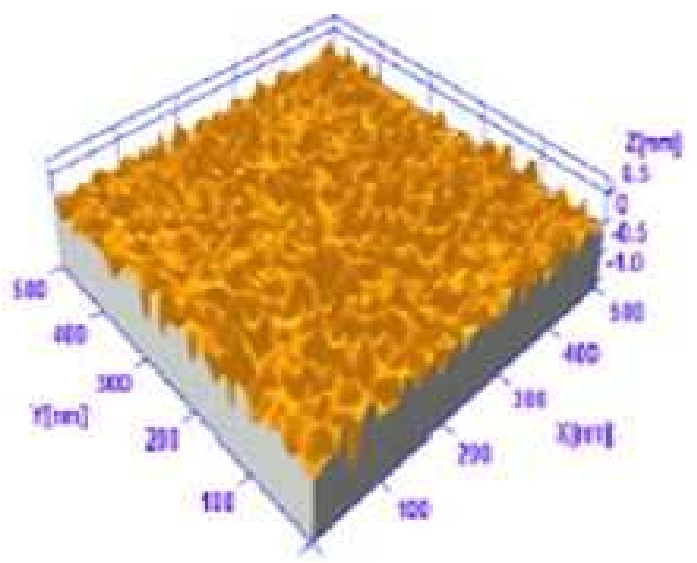

Fig. 4: 3-D image of the same sample [window size = $500 \mathrm{nmX500nm}$.] of Fig. 2

With the advancement of the time of deposition, the density as well as the height increases steadily which can well be observed from our next AFM image of the deposition of nanoparticles at $600 \mathrm{C}$ for $120 \mathrm{secs}$ duration. 


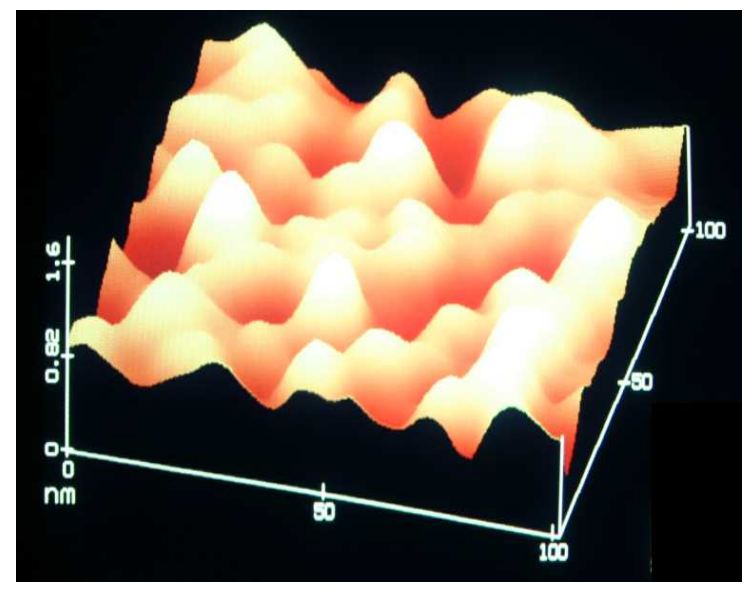

Fig. 5: AFM image of nanoparticles deposited at $600 \square \mathrm{C}$ for $120 \mathrm{sec}$

\section{DISCUSSION}

These nanoparticles have been fabricated by controlling the primary stages of nucleation and growth process. Deposited nanoparticles depend on the pressure, temperature and the deposition time. Nanoparticles may be deposited at different temperature. Here, the deposition temperature was $6000 \mathrm{C}$ and the deposition time was also varied between $30 \mathrm{sec}$ to $120 \mathrm{sec}$. Initially, for small deposition time, the formation of nuclei just starts. So the density was very low. As the deposition time is increased, new atoms will amalgamate on the top of the existing nuclei resulting in enlarged particles. The new nuclei will also form simultaneously. As the deposition time is further raised, the density of nanoparticles will decrease because of agglomeration of smaller particles to form larger clusters. Densities vary from $2 X 1011 / \mathrm{cm} 2$ to $3.5 X 1011 / \mathrm{cm} 2$. In Fig. 4, AFM image of silicon nanoparticles elucidate the density of nanoparticles of about 2X1011/cm2.This figure also shows the 3D image of window 500nmX500nm. It has been proved that the density of nanoparticles as well as their height change with deposition time. The enlarged image extracted from this window shows the shape and size. The sizes of nanodots are in the range of 2-10 $\mathrm{nm}$ and the average height is in the range of 1-3 nm. In Fig. 4, the AFM image of nanoparticles deposited at $6000 \mathrm{C}$ for $120 \mathrm{sec}$ shows the average height of the nanoparticles as $1.6 \mathrm{~nm}$. The size of the nanoparticles also varies with the deposition temperature and duration.

\section{CONCLUSIONS}

Silicon nanoparticles were synthesized using low pressure chemical vapour deposition (LPCVD) method. It has been shown that the height varied between 1-3 nm whereas the densities varied between $2 \times 10^{11} / \mathrm{cm}^{2}$ to $3.5 \times 10^{11} / \mathrm{cm}^{2}$. The size of the nanoparticles also varied between $2-10 \mathrm{~nm}$. Thus, it can be concluded that the height, density as well as the size of the synthesized nanoparticles vary with change in deposition time.

\section{ACKNOWLEDGEMENTS}

We would like to thank Shri Anil Kumar (Scientist, CEERI, Pilani) and Shri Pankaj Bhooshan Agarwal (Scientist, CEERI, Pilani) for giving us the opportunity to carry out the experiment in their laboratory and for their valuable suggestions.

\section{REFERENCES}

[1]. Fu Y, Willander M, Dutta A and Oda S, Superlattice Microstructures 28 (2000) 177

[2]. Nishiguchi K and Oda S, J. Appl. Phys. 88 (2000) 4186

[3]. Fakuda M, Nakagawa K,Miyazaki K, Hirose M, Appl.Phys. Lett. 70 (1997) 2291

[4]. Takahashi Y, Fujiwara A, Yamazaki K, Namatsu H, Kurihara K, Murase K, Abs. Int. Conf. Solid State Devices Materials (1998) 176

[5]. Tiwari S, Rana E, Hanafi H, Hartstein H. Crabb E E, Chan E, Appl. Phys. Lett. 68 (1997) 1377

[6]. Banerjee S, Huang S, Yamanaka T and Oda S, J. Vac. Sci. Technol. B 20 (2002) 1135

[7]. Ostraat M L, De Blauwe J W, Green M L, Bell L D, Brongersma M L, Casperson J, Flagan R C and Atwater H A, Appl. Phys. Lett. 79 (2001) 433

[8]. Tiwari S, Rana F, Chan K, Shi L and Hanafi H, Appl. Phys. Lett. 69 (1996) 1232

[9]. Rassel R M, Kim T, Shen Z, Campbell S A, McMurry P H, J. Vac. Sci. Techn. B, 21,6, (2003) 2441

[10]. Campbell S A, Kortshagen U, Bapat A, Dong Y, Hilchie S and Shen Z, J. of Mat. Oct (2004) 26

[11]. Furukawa S, Kagawa T and Matsumoto N, Solid State Commun. 44 (1982) 927

[12]. Gopireddy D, Takoudis C G, Gamota D, Zhang J and Brazis P W, NSTI Nanotech 2005, NSTI Nanotechnology Conference and Trade Show,2, (2005) 515.

[13]. Nakajima A, Sugita Y, Kawamura K, Tomita H, Yokoyama N, Jpn. J. Appl. Phys. 35 (1996) L189.

[14]. Nakagawa K, Fukuda M, Miyazaki S, Hirose M, MRS Symp. Proc. 452 (1997) 243.

[15]. Mayazaki S, Hamamoto Y, Yoshida E, Ikeda M, Hirose M, Thin Solid Films (2000) 55

[16]. Mazen F, Baron T, Hartmann J M, Semeria M N, Bremond G, MRS Symp. Proc. 737 (2003) F1.9.1

\section{BIOGRAPHIES}

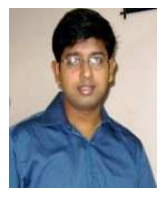

Mr.M.B.Sarkar is currently assistant professor in ECE department of NIT, Agartala. He has Several publications in reputed journals. His area of research interests is RF and Micro Wave Engg., Wireless Communication, Nano Electronics, Semiconductor and Opto Electronic devices. 
Mr.Joydeep Datta is a B.Tech student in ECE dept. at RCC Institute, Kolkata, West Bengal, India. His research interest includes nanodot embedded semiconductor structures and devices

Mr.Diptojyoti Mondal is a B.Tech student in ECE dept. at RCC Institute, Kolkata, West Bengal, India. His research interest includes semiconductor structures and devices.

Mr. Sabyasachi Mukhopadhyay currently is a Research Fellow in Physical Sciences department of IISER, Kolkata. He completed B.Tech in ECE from College of Engineering \& Management, Kolaghat in July,2012. Till now he has 21 numbers of International Journals, International/ National Conference Proceedings with winning the best research paper award once. His areas of research interests are Digital Signal \& Image Processing, Renewable Energy, Semiconductor Physics and Graph Theory. 